MRS. HEIKE CAROLIN HAWERKAMP (Orcid ID : 0000-0002-3990-8230)

Article type : Original Article: Skin and Eye Diseases

\title{
Vemurafenib acts as an aryl hydrocarbon receptor antagonist: Implications for inflammatory cutaneous adverse events
}

Heike C. Hawerkamp ${ }^{a \star}$, Andreas Kislat ${ }^{a \star}$, Peter A. Gerber ${ }^{\mathrm{a}}$, Marius Pollet ${ }^{\mathrm{b}}$, Katharina M. Rolfes ${ }^{b}$, Anatoly A. Soshilov ${ }^{c}$, Michael S. Denison ${ }^{c}$, Afaque A. Momin ${ }^{d}$, Stefan T. Arold $^{d}$, Angeliki Datsi ${ }^{a}$, Stephan A. Braun ${ }^{a}$, Péter Oláh ${ }^{a, e}$, Mario E. Lacouture ${ }^{f}$, Jean Krutmann $^{\mathrm{b}}$, Thomas Haarmann-Stemmann ${ }^{\mathrm{b} \#}$, Bernhard Homey ${ }^{\mathrm{a}}$, and Stephan Meller $^{\text {a\# }}$

aDepartment of Dermatology, Medical Faculty, Heinrich-Heine-University, Duesseldorf, Germany

${ }^{b}$ Leibniz-Research Institute for Environmental Medicine, Duesseldorf, Germany

${ }^{c}$ Department of Environmental Toxicology, University of California, Davis, CA, USA ${ }^{d}$ King Abdullah University of Science and Technology (KAUST), Computational Bioscience Research Center (CBRC), Division of Biological and Environmental Sciences and Engineering (BESE), Thuwal, 23955-6900, Saudi Arabia

${ }^{e}$ Department of Dermatology, Venereology and Oncodermatology, University of Pécs, Pécs, Hungary

${ }^{f}$ Dermatology Service, Department of Medicine, Memorial Sloan-Kettering Cancer Center, New York, NY, USA

This article has been accepted for publication and undergone full peer review but has not been through the copyediting, typesetting, pagination and proofreading process, which may lead to differences between this version and the Version of Record. Please cite this article as doi: 10.1111/all.13972

This article is protected by copyright. All rights reserved. 
${ }^{* \#}$ Equal contribution

Corresponding author:

Stephan Meller, MD

Department of Dermatology, Medical Faculty, Heinrich-Heine-University Duesseldorf Moorenstr. 5

D-40225 Duesseldorf, Germany

Phone: + 492118117853

Fax: + 492118117316

e-mail: Stephan.Meller@uni-duesseldorf.de

Short title: Vemurafenib acts as aryl hydrocarbon receptor antagonist

Conflict of interest statement: The authors declare no potential conflicts of interest.

Keywords: aryl hydrocarbon receptor, drug eruption, lymphocyte activation test, melanoma, vemurafenib

\section{Abbreviations:}

ADR, adverse drug reaction

AhR, aryl hydrocarbon receptor

BRAFi, BRAF protein kinase inhibitor

EGFRi, EGFR inhibitor

h, hours

LAT, lymphocyte activation test

SI, stimulation index

$V I R$, vemurafenib-induced rashes

$\mathrm{XRE}$, xenobiotic response element

\section{Abstract}

Background: In recent years, the BRAF-inhibitor vemurafenib has been successfully established in the therapy of advanced melanoma. Despite its superior efficacy, the use of vemurafenib is limited by frequent inflammatory cutaneous adverse events 
that affect patients' quality of life and may lead to dose reduction or even cessation of anti-tumor therapy. To date, the molecular and cellular mechanisms of vemurafenib-induced rashes have remained largely elusive.

Methods: In this study we deployed immunohistochemistry, RT-qPCR, flow cytometry, lymphocyte activation tests and different cell-free protein-interaction assays.

Results: We here demonstrate that vemurafenib inhibits the downstream signaling of the canonical pathway of aryl hydrocarbon receptor (AhR) in vitro, thereby inducing the expression of proinflammatory cytokines (e.g. TNF) and chemokines (e.g. CCL5). In line with these results we observed an impaired expression of AhR regulated genes (e.g. CYP1A1) and an upregulation of the corresponding proinflammatory genes in vivo. Moreover, results of lymphocyte activation tests showed the absence of drug-specific $\mathrm{T}$ cells in respective patients.

Conclusion: Taken together, we obtained no hint of an underlying sensitization against vemurafenib but found evidence suggesting that vemurafenib enhances proinflammatory responses by inhibition of canonical AhR signaling. Our findings contribute to our understanding of the central role of the AhR in skin inflammation and may point towards a potential role for topical AhR agonists in supportive cancer care.

This article is protected by copyright. All rights reserved. 


\section{Introduction}

The introduction of immune checkpoint-inhibitors (e.g. ipilimumab, pembrolizumab, nivolumab), BRAF- (vemurafenib, dabrafenib) and MEK-inhibitors (cobimetinib, trametinib), has revolutionized the treatment of advanced melanoma. The first of its class BRAF protein kinase inhibitor (BRAFi) vemurafenib received FDA approval in August $2011^{1}$. Although vemurafenib is generally well tolerated, cutaneous adverse events occur in up to $70 \%$ of treated patients ${ }^{2}$ and include inflammatory maculopapular rashes (up to $37 \%)$, photosensitivity $(12 \%)$ or secondary skin tumors $(20 \%)$

1. Cutaneous adverse effects represent a serious threat to patients' therapy adherence and may lead to dose reduction or therapy cessation. Despite clinical relevance, molecular and cellular mechanisms of vemurafenib-associated rashes (VIR) have remained largely elusive. Interestingly, Chapman and coworkers reported that pre-treatment with the pro-inflammatory, anti-cytotoxic $\mathrm{T}$ lymphocyte-associated antigen blocking antibody ipilimumab was associated with a higher frequency (up to $70 \%$ ) of VIRs ${ }^{3}$. The authors proposed that increased numbers of severe grade 3 rashes are the result of hypersensitivity drug reactions to vemurafenib driven by immune checkpoint inhibition. In patients who developed grade 3 rashes vemurafenib therapy was paused until the rash resolved and were successfully retreated with lower doses of vemurafenib ${ }^{3,4}$. These clinical observations point towards either drug hypersensitivity through allergic sensitization or a pharmacological mechanism enhancing cutaneous inflammations. Here, we set out to investigate the underlying molecular mechanisms of VIRs. To this end, we characterized the phenotype of the rashes, performed lymphocyte activation tests (LAT) and investigated potential interactions with the signaling of the aryl 
hydrocarbon receptor (AhR) representing a well-known xenobiotic receptor and central regulator of inflammatory processes ${ }^{5-7}$.

\section{Material and Methods}

\subsection{Human samples}

Punch biopsies were obtained from patients with VIRs after obtaining informed consent. For skin explants, punch biopsies were taken from healthy skin. Buffy coats from healthy donors were obtained from the Institute of Hemostasis and Transfusion Medicine, University Hospital Duesseldorf. The guidelines of the Declaration of Helsinki were deployed and the study was authorized by the local ethics committee (No: 1928).

\subsection{Animal samples and preparation of cytosol}

Hartley guinea pigs were obtained from Charles River (Wilmington, DE, USA). Guinea pig hepatic cytosol was prepared as described ${ }^{8}$ and stored properly until use. All animal protocols were approved by the Institutional Animal Care and Use Committee of the University of California, Davis.

\subsection{Culture of human cells and explants}

The culture of ex vivo explants, keratinocytes and T cells is described in the supplemental methods.

The tested concentrations of vemurafenib (up to $100 \mu \mathrm{M}$ ) ${ }^{9,10}$ and dabrafenib ${ }^{10,11}$ correspond to published studies. Cell viability was confirmed using 3-(4,5dimethylthiazol-2-yl)-2,5-diphenyltetrazolium bromide (not shown). 


\subsection{RNA extraction and qPCR}

Biopsies were homogenized in TRIzol® using a POLYTRON PT2500E (KINEMATICA AG, Luzern, Switzerland). RNA was isolated using RNeasy Mini Kit (Qiagen, Hilden, Germany) following the manufacturer's instructions, reverse transcribed into cDNA and analyzed by quantitative real-time PCR (ABI PRISM ${ }^{\circledR}$ 7000 Sequence Detection System/ QuantStudio 6 Flex, Thermo Fisher Scientific) ${ }^{12}$.

\subsection{Statistics}

Statistical significances were assessed with Mann-Whitney $U$ tests or Kruskal-Wallis test with Dunn's post correction and calculated using GraphPad Prism 5.03 (GraphPad software, Inc., La Jolla, CA, USA). Statistical significances were depicted as follows: ${ }^{*} p<0.05,{ }^{* *} p<0.01$ and ${ }^{* * *} p<0.001$.

Additional methods are described in the supplemental methods.

\section{Results}

\subsection{Vemurafenib-induced inflammatory rashes are characterized by a dense lymphohistiocytic infiltrate}

Patients ( $n=5 ; 67-76$ years) with VIRs and healthy controls $(n=5 ; 52-74$ years) were included in our analysis. Patients presented with a generalized maculopapular rash with small papules and macules without scaling (Figure 1A). Histopathologic evaluation of lesional skin biopsies demonstrated a superficial dermatitis without epidermal changes, with mild spongiosis or subtle vacuolar interface changes. Immunohistochemistry revealed a lymphohistiocytic infiltrate with equally distributed 
$\mathrm{CD}^{+}$and $\mathrm{CD} 8^{+} \mathrm{T}$ cells (Figure 1B). We did not observe any prominent infiltrates of eosinophils, neutrophils or mast cells.

\subsection{Vemurafenib-induced inflammatory rashes are characterized by a predominant $T_{H} 1$ - signature}

We next analyzed the expression of signature cytokines in lesional skin (VIR, $n=4-5)$ compared to healthy controls (HS, $n=5)$. Our analyses revealed a significant induction of $T_{H} 1$-associated cytokine IFNG, IFN- $\gamma$-induced chemokines CXCL9-11, CCL5 (Figure 1C), and a significant upregulation of homeostatic chemokines CCL27 and CXCL14 (Supplementary Figure S1B). Moreover, pro-inflammatory cytokines and chemokines such as CCL2 and IL1B were found to be upregulated (Figure 1C, Supplementary Figure S1B). Although, we observed increased expression levels of the $\mathrm{T}_{\mathrm{H}} 2$-associated chemokines CCL1, CCL17, CCL18, and CCL22, no relevant regulation was detected for IL4. Furthermore, IL17A or IFNA were not induced in lesional skin (Figure 1C, Supplementary Figure S1B). Taken together, we observed a predominant upregulation of $T_{H} 1$-associated chemokines.

\subsection{Vemurafenib induces inflammatory cytokines and chemokines in vitro}

Next, we analyzed the recruitment pathways directing leukocyte subsets to sites of vemurafenib-induced cutaneous inflammation. We performed comprehensive analyses of cytokine and chemokine expression in whole skin explants, primary human keratinocytes, and T cells treated with vemurafenib in vitro. In skin explants vemurafenib alone altered the expression of CCL5 and CCL18, which tended to be upregulated, mimicking the expression profile of VIRs (Figure 2A). In keratinocytes

vemurafenib induced a significant expression of TNF and CCL5 (Figure 2A). In T 
cells, an early upregulation of IFNG after $6 \mathrm{~h}$ was observed, and after $24 \mathrm{~h}$ transcription and protein levels were increased (Figure 2B). Further, IL17A was upregulated after $6 \mathrm{~h}$ and $24 \mathrm{~h}$ of vemurafenib treatment, yet at overall low expression levels (Supplementary Figure S2A). IL4 expression was induced by vemurafenib at negligible levels (Supplementary Figure S2B).

\subsection{Absence of circulating drug-specific $T$ cells in patients with vemurafenib- induced rashes}

To discriminate between allergic or non-allergic pharmacologic effects, we performed LATs with leukocytes obtained from patients suffering from VIRs $(n=4)$. To distinguish between allergic and non-allergic patients, Beeler et al. suggested a stimulation index (SI) cutoff value of $2{ }^{13}$. Using this threshold, we did not detect vemurafenib-specific T cells in any patient. Hence, VIRs are rather a result of a nonallergic, pharmacologic mechanism than a result of a specific sensitization against the drug (Figure 2C and 2D).

\subsection{The structure of vemurafenib is compatible with binding to AhR in silico}

To identify non-allergic pharmacologic mechanisms as potential cause of VIRs we focused on the well-known xenobiotic receptor AhR that binds and regulates the metabolism of drugs and other xenobiotics. Recent findings indicate that the loss of the AhR results in cutaneous inflammation ${ }^{14}$. Several protein kinase inhibitors modulate AhR activity in an agonistic or antagonistic manner ${ }^{15}$. The AhR ligand indirubin also binds kinase domains in the same ATP-competitive way as does vemurafenib ${ }^{16-19}$, suggesting some level of cross-reactivity between ligands for AhR and kinases. AhR binds to $\left[{ }^{3} \mathrm{H}\right] \mathrm{TCCD}$ and other aromatic hydrocarbon ligands (PAS- 
B, residues 277-393) through its second PAS domain ${ }^{20}$. The dimensions and molecular characteristics of vemurafenib are within the range of characteristics displayed by bona fide AhR ligands (i.e. $\sim 14 \times 12 \times 5 \AA$ planar, hydrophobic ring structures with some hydrophilic moieties ${ }^{20}$ ). To computationally probe vemurafenib binding to the AhR PAS-B domain, we inferred the atomic structure of PAS-B based on $\sim 26 \%$ identical known PAS-B domain structures. The structural models of PAS-B showed a hydrophobic cavity, in shape and stereochemical character similar to the ligand binding pocket of the HIF-2 $\alpha$ PAS domain (PDB 4xt2), suggesting that PAS-B binds ligands in the same way as does HIF-2a PAS. Using a conservative in silico docking approach that allowed ligand flexibility but kept the protein structure rigid, we observed that indirubin and vemurafenib can be accommodated in the PAS-B pocket without steric clashes (Figure $3 \mathrm{~A}$ and $3 \mathrm{~B}$ ). The calculated binding energies of indirubin and vemurafenib were very similar $(-8.29$ and $-7.91 \mathrm{kcal} / \mathrm{mol}$, respectively, corresponding to dissociation constants $K_{d}$ of 1-3 $\mu \mathrm{M}$ ) supporting that vemurafenib may be a relevant AhR binding partner.

\subsection{Vemurafenib is an AhR antagonist}

To test the hypothesis that vemurafenib is an AhR ligand, we used human HepG2 hepatoma cells, stably transfected with a luciferase reporter gene driven by two AhR binding-sites ${ }^{21}$. HepG2 cells were exposed to vemurafenib alone or in combination with the AhR agonist benzo[a]pyrene (BaP). Basal luciferase reporter gene activity was slightly reduced following incubation with $40 \mu \mathrm{M}$ vemurafenib (data not shown). Similarly, incubation with $40 \mu \mathrm{M}$ vemurafenib markedly reduced the level of AhR-dependent luciferase activity by BaP, suggesting that vemurafenib is acting as an AhR antagonist (Figure 3C). By contrast, luciferase assays did not show any 
inhibitory effect of dabrafenib or encorafenib on AhR activity in relevant concentrations (up to $1 \mu \mathrm{M}$ ) ${ }^{10,22}$ (Supplementary Figure S3A/B). Dabrafenib and encorafenib even significantly increased AhR-dependent luciferase activity at low concentrations of $0.1 \mu \mathrm{M}$ (in absence of $\mathrm{BaP}$ ). The multikinase inhibitor sorafenib showed a slight trend towards AhR inhibition at pharmacological concentrations of $10 \mu \mathrm{M}^{23}$ in presence of BaP (Supplementary Figure S3C). A similar inhibitory trend was seen for the novel BRAF inhibitor PLX8394 (Supplementary Figure S3D).

To determine the mechanism by which vemurafenib reduces AhR-dependent gene expression, we examined its ability to directly bind to AhR. Hydroxyapatite ligand binding analysis using guinea pig hepatic cytosolic AhR revealed that vemurafenib competitively inhibits $\left[{ }^{3} \mathrm{H}\right] \mathrm{TCDD}$, a high affinity AhR ligand, from binding to AhR. This inhibition occurs in a vemurafenib concentration-dependent manner; with maximum competitive inhibition observed at $40 \mu \mathrm{M}$ and hence in line with our micromolar affinities as calculated in silico (Figure 3D). While this analysis confirmed that vemurafenib is a direct ligand for AhR, it does not provide any information as to whether vemurafenib is binding as an AhR agonist or antagonist. To address this question, we utilized a gel retardation analysis to assess the ability of a chemical to stimulate AhR transformation and DNA binding, a characteristic of AhR agonists. Although incubation of guinea pig hepatic cytosol with vemurafenib did not stimulate AhR transformation or DNA binding, it did produce a concentration-dependent decrease in TCDD-stimulated AhR transformation and DNA binding (Figure 3E). Ligand and DNA binding analyses confirmed the ability of vemurafenib to act as an AhR antagonist (i.e. it exhibits no AhR agonist activity). Against the background of previous studies that have demonstrated species-specific differences in AhR ligand 
binding specificity, respective DNA binding analysis studies were repeated using in vitro synthesized human AhR and ARNT (the AhR dimerization partner required for AhR DNA binding) ${ }^{5,24}$. Similar to the results obtained with guinea pig hepatic cytosolic AhR, vemurafenib antagonized the ability of TCDD to stimulate transformation and DNA binding of the human AhR (Figure 3F). As expected, vemurafenib treatment inhibited basal as well as BaP-induced nuclear translocation of AHR in human keratinocytes (Figure 3G). Taken together, these results confirm the ability of vemurafenib to act as an AhR antagonist.

\subsection{Vemurafenib impairs CYP1A1 expression in vitro, ex vivo and in vivo}

In order to prove the AhR antagonism of vemurafenib in vivo and in vitro, we assessed the expression of AhR and AhR-related genes in lesional skin of VIRs compared to healthy controls as well as in skin explants, keratinocytes, and T cells. As an additional control we analyzed papulopustular rashes of cancer patients treated with epidermal growth factor receptor (EGFR) inhibitors, which we have demonstrated to be caused by non-allergic, pharmacologic mechanisms ${ }^{25}$.

$A H R$ was significantly upregulated in VIRs (Supplementary Figure S4A). In contrast, no regulation of $A H R$ was observed in EGFR-inhibitor (EGFRi) induced rashes or healthy controls (Supplementary Figure S4C). Next, we assessed a dysregulation of AhR signaling and analyzed the endpoints of the canonical and noncanonical pathway of AhR signaling with two representatives: Prostaglandinendoperoxide synthase 2 (COX2), and Cytochrome P450 (CYP) 1A1. COX2 was significantly upregulated in VIRs as compared to healthy controls (Supplementary Figure S4B). CYP1A1 gene expression was significantly downregulated in VIRs 
compared to healthy controls (Figure 4A). In line with these results CYP1A1 protein expression was found to be reduced in lesional skin (Figure 4B). Furthermore, we observed a downregulation of CYP1A1 after stimulation with vemurafenib in skin explants, keratinocytes, and T cells in vitro (Figure 4C). Vemurafenib did not only reduce the basal CYP1A1 expression but also it decreases it when skin explants have been stimulated with the AhR agonist FICZ (Figure 4C).

Comparing vemurafenib to dabrafenib in terms of $\mathrm{T}$ cell stimulation, we found that dabrafenib did not induce IFN-y and IL-17 at gene or protein level in the pharmacological relevant levels up to $1 \mu \mathrm{M}$ (Figure $4 \mathrm{~F} / \mathrm{G}$ ). Furthermore, dabrafenib did not affect CYP1A1 gene expression and IL-22 protein expression in CD4 ${ }^{+} \mathrm{T}$ cells (Figure 4F/G).

\section{Discussion}

Despite its clinical efficacy, vemurafenib therapy is associated with a wide range of adverse events including arthralgia, fatigue, and skin toxicities ${ }^{26}$. Cutaneous side effects are predominant and occur in up to $70 \%$ of the patients ${ }^{2}$. Four different cutaneous reaction patterns have been described: i) inflammatory reactions, ii) hair/ nail changes, iii) melanocytic disorders/ proliferations, and iv) keratinocytic proliferations ${ }^{27}$. Proliferative alterations have been systematically analyzed and are contributed to a pharmacologic, paradoxical activation of the RASRAF-MEK-ERK signaling pathway ${ }^{28}$. This concept is supported by clinical observations showing that BRAFi-induced proliferative lesions regress under concurrent administration of MEK-inhibitors (MEKi) ${ }^{29,30}$. By contrast, co- 
administration of MEKi results in a slight increase in incidence and severity of inflammatory rashes (35\% for vemurafenib plus placebo versus $39 \%$ for vemurafenib plus cobimetinib) ${ }^{29}$. These observations support the hypothesis that inflammatory rashes and proliferative alterations are caused by different mechanisms.

From a clinical perspective, VIRs present as maculopapular drug exanthemas, pointing towards a $\mathrm{T}$ cell-mediated hypersensitivity reaction ${ }^{31}$. Indeed, immunohistochemical analyses of lesional skin revealed a lymphohistiocytic infiltrate. However, we did not observe prominent infiltrates of eosinophils. Our analyses of cytokines revealed a $T_{H} 1$-signature with an induction of dendritic cell (DC)associated as well as T cell-derived inflammatory mediators, such as $T N F, I L 1 B$, and IFNG, and the $\mathrm{T}_{\mathrm{H}} 1$-associated chemokines CXCL9-11. In addition, the increased expression of CCL27 and CXCL14 facilitates the recruitment of $\mathrm{T}$ cells as well as macrophages ${ }^{32}$. The upregulation of $I L 1 B$ indicates an activation of the inflammasome in macrophages and/or other cells of the innate immune system. Conversely, we did not detect a relevant induction of $\mathrm{T}_{\mathrm{H}}$ 2-cytokines, IFNA or $\mathrm{T}_{\mathrm{H}}$ 17associated cytokines. To test our hypothesis that VIRs are caused rather by pharmacologic than allergic mechanisms, we performed LATs of blood samples obtained from patients with VIRs. Whereas stimulated T cells expressed IFN- $\gamma$ RNA and protein, LATs failed to detect vemurafenib-specific T cells in our collective. To conclude, the negligible numbers of eosinophils, the absence of an allergyassociated $\mathrm{T}_{\mathrm{H}} 2$ signature, and the absence of drug-specific $\mathrm{T}$ cells, indicate that VIRs are caused by non-allergic pharmacological reactions rather than a drugspecific sensitization. This hypothesis is further supported by an observed induction of chemokines, including CCL2, CCL5, CCL27 and CXCL14, which also play a 
central role for the pathogenesis of non-allergic, pharmacologic, papulopustular rashes induced by $E G F R i^{25}$. In contrast, non-immediate allergic drug reactions are associated with a $T_{H} 1$ chemokine signature ${ }^{33,34}$. To assess whether the induction of inflammation is caused by a direct pharmacological effect of vemurafenib on skin or by indirect, systemic effects, skin explants were stimulated with vemurafenib ex vivo. In line with the cytokine-chemokine signature observed in patient samples in vivo, we found a marked induction of TNFA and CCL5. Respective in vitro analyses in human keratinocytes and $\mathrm{T}$ cells showed similar results, indicating that structural cells and immune cells are targets of vemurafenib and central regulators of vemurafenibinduced inflammation in the skin.

Although, vemurafenib and dabrafenib are both potent inhibitors of the BRAFkinase, reported incidences for inflammatory rashes are significantly higher for vemurafenib ( $41 \%$ vs. $30 \%)$, suggesting that these rashes are rather not caused by a class-specific effect of BRAFi but by another, so far unknown vemurafenib-specific mechanism ${ }^{35}$. A putative target for vemurafenib is the xenobiotic receptor AhR, which has been shown to be modulated by several other protein kinase inhibitors in an either agonistic or antagonistic manner ${ }^{7,15,36-38}$. Interestingly, AhR is highly expressed in skin and other barrier organs ${ }^{5}$. Upon binding of an agonistic ligand, AhR stimulates expression of a battery of genes in a tissue- and cell-specific fashion 5,24. Activation of the canonical signaling pathway involves ligand-dependent translocation of AhR into the nucleus, where it forms a dimer with the AhR nuclear translocator (ARNT) resulting in the binding to an upstream regulatory region of respective target genes ${ }^{5}$. In addition, AhR activation leads to stimulation of noncanonical signaling pathways, which may trigger pro-inflammatory responses $5,39,40$. 
As recently postulated, canonical and non-canonical AhR signaling pathways are tightly balanced in healthy skin thereby contributing to skin homeostasis ${ }^{41}$. In fact, our analysis revealed a decreased number of CYP1A1 transcripts (as marker for canonical AhR signaling) in vemurafenib-induced rashes, which was paralleled by elevated mRNA levels of COX2 (as marker for non-canonical AhR signaling). Of note, a recent study using an imiquimod mouse model of psoriasiform dermatitis indicates that loss of AhR results in exaggerated cutaneous inflammation ${ }^{42}$. Accordingly, treatment of wild-type mice with AhR agonists attenuated psoriasiform skin inflammation ${ }^{42}$. AhR activation by coal tar was shown to reduce proinflammatory responses and restore epidermal barrier functions in a 3D in vitro model of atopic dermatitis ${ }^{43}$. Furthermore, inflammatory responses in lipopolysaccharide-challenged AhR-deficient mice are characterized by induction of TNF- $\alpha$, IL-1 $\beta$, IL-6, IL-18 and IFN- $\gamma^{44}$, a cytokine pattern closely resembling the one observed in VIRs.

Di Meglio et al. identified that deficiency or antagonism of the nonhematopoietic skin compartment is necessary and sufficient for the development of skin inflammation ${ }^{42}$. Their results ruled out a central role for DCs or macrophages but did not exclude a possible involvement of $\mathrm{T}$ or $\mathrm{B}$ cells in AhR-mediated skin inflammation. In line with these results we did not observe significant effects of vemurafenib on DCs (not shown) but demonstrate that vemurafenib downregulates the AhR prototype target gene CYP1A1 in keratinocytes and T cells in vitro as well as skin explants ex vivo. These findings are supported by the observation that CYP1A1 gene expression was down-modulated in VIRs in vivo. By contrast, CYP1A1 was not altered in EGFRi-associated rashes as compared to healthy 
donors. This indicates that VIRs are likely triggered by disturbance of canonical but not non-canonical AhR signaling that involves the c-Src/EGFR/ERK pathway ${ }^{40}$. Our hypothesis that vemurafenib acts as a potent AhR antagonist is further supported by reporter gene analyses, showing that vemurafenib treatment inhibited both basal as well as BaP-induced activity of the AhR-dependent luciferase construct. In fact, competitive ligand-binding assays and determination of DNA-bound AhR/ARNT protein provided evidence that vemurafenib is an antagonistic ligand of AhR. In contrast to a recent study on MCF-7 breast cancer cells, reporting that vemurafenib treatment induces nuclear translocation of $\mathrm{AhR}$ (Corre et al. ${ }^{45}$ ), our data clearly show that the BRAF inhibitor retained AhR in the cytosol of solvent- as well as BaPtreated keratinocytes and thus behaves as a pure antagonist. The molecular mechanism responsible for this apparent cell-specific differences remains to be elucidated. As opposed to vemurafenib, dabrafenib did not interfere with AhR and dabrafenib-treated patients develop cutaneous rashes at lower incidences ${ }^{46,47}$. Taking in account our hitherto results it is also not surprising that immunostimulatory immune-checkpoint inhibitors such as ipilimumab, nivolumab or pembrolizumab, can aggravate the severity of vemurafenib-induced rashes ${ }^{3,4,48}$. Up to $70 \%$ of patients treated with vemurafenib develop a drug eruption. The initial trigger remains unclear. Unknown factors such as infectious triggers, environmental /food related xenobiotics or an individual genetic predisposition may favor the spontaneous onset of the observed rashes.

Taken together, our results indicate that vemurafenib-induced inflammatory rashes are caused by a pharmacologic mechanism rather than a specific sensitization against the drug. Moreover, our results imply that this mechanism is not 
a class-specific, direct inhibition of BRAF, but rather a vemurafenib-specific inhibition of the AhR. Interestingly, Smith et al., identified a naturally derived topical AhR agonist called tapinarof (GSK2894512) capable of inducing CYP1A1 expression together with an improvement of murine AhR-mediated imiquimod-induced psoriasiform skin lesions ${ }^{49}$. Against this background, we hypothesize that activation of AhR for instance via an AhR agonist-rich diet, (e.g. brassica vegetables containing indole-3-carbinol), or via topical application of non-toxic AhR ligands may represent a novel strategy to prevent or treat VIRs ${ }^{50}$.

\section{Acknowledgments}

We thank Paul Chapman and Katja Schindler for providing clinical images and proofreading the manuscript and Katarina Gradin and Lorenz Poellinger for generously providing the XRE-HepG2 reporter cell line. The research by Stefan T. Arold and Afaque A. Momin was supported by the King Abdullah University of Science and Technology (KAUST) through the Award No. FCC/1/1976-25, from the Office of Sponsored Research (OSR). Mario E. Lacouture is funded in part through the NIH/NCl Cancer Center Support Grant P30 CA008748. Thomas HaarmannStemmann and Katharina M. Rolfes were supported by grants of the Deutsche Forschungsgemeinschaft (HA 7346/2-1) and the Jürgen Manchot Foundation.

This article is protected by copyright. All rights reserved. 


\section{Author contributions}

H.C.H., A.K. and P.A.G. designed, performed and analyzed most of the experiments. M.P. helped in performing the luciferase assays and implemented the AhR translocation assay. K.M.R. conducted the EROD assays. A.A.S and M.S.D. designed and performed the AhR binding experiments. A.A.M and S.T.A. conducted the in silico computational modelling. A.D. performed flow cytometry experiments. S.A.B. did the immunohistochemistry experiments and helped with microscopy. P.O., M.E.L. and J.K. provided suggestions and discussions throughout the study. S.M. supervised the study and wrote the manuscript together with H.C.H., A.K., P.A.G., T.H. and B.H.

\section{References}

1. Chapman PB, Hauschild A, Robert C, et al. Improved survival with vemurafenib in melanoma with BRAF V600E mutation. N Engl J Med. 2011;364(26):2507-2516.

2. Manousaridis I, Mavridou S, Goerdt S, Leverkus M, Utikal J. Cutaneous side effects of inhibitors of the RAS/RAF/MEK/ERK signalling pathway and their management. $J$ Eur Acad Dermatol Venereol. 2013;27(1):11-18.

3. Harding JJ, Pulitzer M, Chapman PB. Vemurafenib sensitivity skin reaction after ipilimumab. N Engl J Med. 2012;366(9):866-868.

4. Minor DR, Rodvien R, Kashani-Sabet M. Successful desensitization in a case of StevensJohnson syndrome due to vemurafenib. Melanoma Res. 2012;22(5):410-411.

5. Esser C, Rannug A. The aryl hydrocarbon receptor in barrier organ physiology, immunology, and toxicology. Pharmacol Rev. 2015;67(2):259-279.

6. Jin UH, Lee SO, Safe S. Aryl hydrocarbon receptor (AHR)-active pharmaceuticals are selective AHR modulators in MDA-MB-468 and BT474 breast cancer cells. J Pharmacol Exp Ther. 2012;343(2):333-341.

7. Zhao B, Degroot DE, Hayashi A, He G, Denison MS. CH223191 is a ligand-selective antagonist of the Ah (Dioxin) receptor. Toxicol Sci. 2010;117(2):393-403.

8. Denison MS, Rogers JM, Rushing SR, Jones CL, Tetangco SC, Heath-Pagliuso S. Analysis of the aryl hydrocarbon receptor (AhR) signal transduction pathway. Curr Protoc Toxicol. 2002; Chapter 4:Unit4 8.

9. da Rocha Dias S, Salmonson T, van Zwieten-Boot B, et al. The European Medicines Agency review of vemurafenib (Zelboraf(R)) for the treatment of adult patients with BRAF V600 mutation-positive unresectable or metastatic melanoma: summary of the scientific assessment of the Committee for Medicinal Products for Human Use. Eur J Cancer. 2013;49(7):1654-1661.

This article is protected by copyright. All rights reserved. 
10. Yaktapour N, Meiss F, Mastroianni J, et al. BRAF inhibitor-associated ERK activation drives development of chronic lymphocytic leukemia. J Clin Invest. 2014;124(11):5074-5084.

11. Falchook GS, Long GV, Kurzrock R, et al. Dabrafenib in patients with melanoma, untreated brain metastases, and other solid tumours: a phase 1 dose-escalation trial. Lancet (London, England). 2012;379(9829):1893-1901.

12. Homey $B$, Wang $W$, Soto $\mathrm{H}$, et al. Cutting edge: the orphan chemokine receptor $\mathrm{G}$ proteincoupled receptor-2 (GPR-2, CCR10) binds the skin-associated chemokine CCL27 (CTACK/ALP/ILC). J Immunol. 2000;164(7):3465-3470.

13. Beeler A, Zaccaria L, Kawabata T, Gerber BO, Pichler WJ. CD69 upregulation on T cells as an in vitro marker for delayed-type drug hypersensitivity. Allergy. 2008;63(2):181-188.

14. Abel J, Haarmann-Stemmann T. An introduction to the molecular basics of aryl hydrocarbon receptor biology. Biological chemistry. 2010;391(11):1235-1248.

15. Frauenstein K, Tigges J, Soshilov AA, et al. Activation of the aryl hydrocarbon receptor by the widely used Src family kinase inhibitor 4-amino-5-(4-chlorophenyl)-7(dimethylethyl)pyrazolo[3,4-d]pyrimidine (PP2). Archives of toxicology. 2014.

16. Leclerc $S$, Garnier M, Hoessel R, et al. Indirubins inhibit glycogen synthase kinase-3 beta and CDK5/p25, two protein kinases involved in abnormal tau phosphorylation in Alzheimer's disease. A property common to most cyclin-dependent kinase inhibitors? J Biol Chem. 2001;276(1):251-260.

17. Marko D, Schatzle S, Friedel A, et al. Inhibition of cyclin-dependent kinase 1 (CDK1) by indirubin derivatives in human tumour cells. Br J Cancer. 2001;84(2):283-289.

18. Karoulia Z, Wu Y, Ahmed TA, et al. An Integrated Model of RAF Inhibitor Action Predicts Inhibitor Activity against Oncogenic BRAF Signaling. Cancer Cell. 2016;30(3):485-498.

19. Mapelli M, Massimiliano L, Crovace $C$, et al. Mechanism of CDK5/p25 binding by CDK inhibitors. J Med Chem. 2005;48(3):671-679.

20. Fujii-Kuriyama Y, Mimura J. Molecular mechanisms of AhR functions in the regulation of cytochrome P450 genes. Biochem Biophys Res Commun. 2005;338(1):311-317.

21. Gradin K, Wilhelmsson A, Poellinger L, Berghard A. Nonresponsiveness of normal human fibroblasts to dioxin correlates with the presence of a constitutive xenobiotic response element-binding factor. J Biol Chem. 1993;268(6):4061-4068.

22. Delord J-P, Robert C, Nyakas M, et al. Phase I Dose-Escalation and -Expansion Study of the BRAF Inhibitor Encorafenib (LGX818) in Metastatic \&It;em\&gt;BRAF\&lt;/em\&gt;-Mutant Melanoma. Clinical Cancer Research. 2017;23(18):5339.

23. Mai H, Huang J, Zhang $\mathrm{Y}$, et al. In-vivo relation between plasma concentration of sorafenib and its safety in Chinese patients with metastatic renal cell carcinoma: a single-center clinical study. Oncotarget. 2017;8(26):43458-43469.

24. Denison MS, Soshilov AA, He G, DeGroot DE, Zhao B. Exactly the same but different: promiscuity and diversity in the molecular mechanisms of action of the aryl hydrocarbon (dioxin) receptor. Toxicol Sci. 2011;124(1):1-22.

25. Lichtenberger BM, Gerber PA, Holcmann M, et al. Epidermal EGFR controls cutaneous host defense and prevents inflammation. Sci Trans/ Med. 2013;5(199):199ra111.

26. Sosman JA, Kim KB, Schuchter L, et al. Survival in BRAF V600-mutant advanced melanoma treated with vemurafenib. N Engl J Med. 2012;366(8):707-714.

27. Rinderknecht JD, Goldinger SM, Rozati S, et al. RASopathic skin eruptions during vemurafenib therapy. PLoS One. 2013;8(3):e58721.

28. Hatzivassiliou G, Song K, Yen I, et al. RAF inhibitors prime wild-type RAF to activate the MAPK pathway and enhance growth. Nature. 2010;464(7287):431-435.

29. Larkin J, Ascierto PA, Dreno B, et al. Combined vemurafenib and cobimetinib in BRAFmutated melanoma. N Engl J Med. 2014;371(20):1867-1876.

30. Chen FW, Tseng D, Reddy S, Daud Al, Swetter SM. Involution of eruptive melanocytic nevi on combination BRAF and MEK inhibitor therapy. JAMA Dermatol. 2014;150(11):1209-1212.

This article is protected by copyright. All rights reserved. 
31. Hoetzenecker W, Nageli M, Mehra ET, et al. Adverse cutaneous drug eruptions: current understanding. Semin Immunopathol. 2016;38(1):75-86.

32. David BA, Kubes P. Exploring the complex role of chemokines and chemoattractants in vivo on leukocyte dynamics. Immunological reviews. 2019;289(1):9-30.

33. Fernandez TD, Mayorga C, Torres MJ, et al. Cytokine and chemokine expression in the skin from patients with maculopapular exanthema to drugs. Allergy. 2008;63(6):712-719.

34. Meller S, Gerber PA, Kislat A, et al. Allergic sensitization to pegylated interferon-alpha results in drug eruptions. Allergy. 2015;70(7):775-783.

35. Welsh SJ, Corrie PG. Management of BRAF and MEK inhibitor toxicities in patients with metastatic melanoma. Ther Adv Med Oncol. 2015;7(2):122-136.

36. Korashy HM, Anwar-Mohamed A, Soshilov AA, Denison MS, El-Kadi AO. The p38 MAPK inhibitor SB203580 induces cytochrome P450 $1 \mathrm{~A} 1$ gene expression in murine and human hepatoma cell lines through ligand-dependent aryl hydrocarbon receptor activation. Chem Res Toxicol. 2011;24(9):1540-1548.

37. Reiners JJ, Jr., Lee JY, Clift RE, Dudley DT, Myrand SP. PD98059 is an equipotent antagonist of the aryl hydrocarbon receptor and inhibitor of mitogen-activated protein kinase kinase. Mol Pharmacol. 1998;53(3):438-445.

38. Mezrich JD, Nguyen LP, Kennedy G, et al. SU5416, a VEGF receptor inhibitor and ligand of the AHR, represents a new alternative for immunomodulation. PLoS One. 2012;7(9):e44547.

39. Matsumura $F$. The significance of the nongenomic pathway in mediating inflammatory signaling of the dioxin-activated Ah receptor to cause toxic effects. Biochem Pharmacol. 2009;77(4):608-626.

40. Haarmann-Stemmann T, Bothe H, Abel J. Growth factors, cytokines and their receptors as downstream targets of arylhydrocarbon receptor (AhR) signaling pathways. Biochem Pharmacol. 2009;77(4):508-520.

41. Haarmann-Stemmann T, Esser C, Krutmann J. The Janus-Faced Role of Aryl Hydrocarbon Receptor Signaling in the Skin: Consequences for Prevention and Treatment of Skin Disorders. J Invest Dermatol. 2015;135(11):2572-2576.

42. Di Meglio P, Duarte JH, Ahlfors $\mathrm{H}$, et al. Activation of the aryl hydrocarbon receptor dampens the severity of inflammatory skin conditions. Immunity. 2014;40(6):989-1001.

43. van den Bogaard EH, Bergboer JG, Vonk-Bergers $M$, et al. Coal tar induces AHR-dependent skin barrier repair in atopic dermatitis. J Clin Invest. 2013;123(2):917-927.

44. Sekine H, Mimura J, Oshima M, et al. Hypersensitivity of aryl hydrocarbon receptor-deficient mice to lipopolysaccharide-induced septic shock. Molecular and cellular biology. 2009;29(24):6391-6400.

45. Corre S, Tardif N, Mouchet N, et al. Sustained activation of the Aryl hydrocarbon Receptor transcription factor promotes resistance to BRAF-inhibitors in melanoma. Nature communications. 2018;9(1):4775.

46. Hauschild A, Grob JJ, Demidov LV, et al. Dabrafenib in BRAF-mutated metastatic melanoma: a multicentre, open-label, phase 3 randomised controlled trial. Lancet. 2012;380(9839):358365.

47. Sanlorenzo M, Choudhry A, Vujic I, et al. Comparative profile of cutaneous adverse events: BRAF/MEK inhibitor combination therapy versus BRAF monotherapy in melanoma. J Am Acad Dermatol. 2014;71(6):1102-1109 e1101.

48. Imafuku K, Yoshino K, Ymaguchi K, Tsuboi S, Ohara K, Hata H. Nivolumab therapy before vemurafenib administration induces a severe skin rash. J Eur Acad Dermatol Venereol. 2016.

49. Smith SH, Jayawickreme C, Rickard DJ, et al. Tapinarof Is a Natural AhR Agonist that Resolves Skin Inflammation in Mice and Humans. J Invest Dermatol. 2017;137(10):2110-2119.

50. Benson JM, Shepherd DM. Dietary ligands of the aryl hydrocarbon receptor induce antiinflammatory and immunoregulatory effects on murine dendritic cells. Toxicol Sci. 2011;124(2):327-338.

This article is protected by copyright. All rights reserved. 


\section{Figures}

Figure 1: Clinical, histologic and molecular characterization of vemurafenibinduced skin rashes. (A), Representative patient with generalized maculopapular rash. (B), Hematoxylin and eosin (HE) stain, Giemsa stain and immunohistochemical analysis of CD1a, CD68, CD4 and CD8 in lesional skin of one representative patient. (C), semi-quantitative PCR analysis of cytokine and chemokine expression in healthy skin (HS, $n=5)$ compared to lesional skin of vemurafenib-induced rashes (VIR, $n=4$ 5). qPCR-values are shown as relative units compared to 18S rRNA expression. Data are presented as single values and median. Mann-Whitney $U$ test was used to evaluate significant differences $\left({ }^{*} p<0.05,{ }^{* *} p<0.01\right)$.

Figure 2: Vemurafenib induces cytokines and chemokines in skin explants, keratinocytes and $\mathbf{T}$ cells, whereas it does not sensitize $\mathbf{T}$ cells. $(A, B)$, Skin explants $(n=6)$, keratinocytes $(n=14-15)$ and total T cells $(n=9-14)$ were treated with vemurafenib [10; $40 \mu \mathrm{M}]$. qPCR-values are shown as mean + SEM of fold change normalized to $18 \mathrm{~S}$ rRNA expression compared to DMSO. (B), IFN-y expression of $\mathrm{CD}^{+} / \mathrm{CD}^{+} \mathrm{T}$ cells $(\mathrm{n}=7)$ after treatment, represented as single values and mean. $(C, D)$, Analysis of $\mathrm{CD}{ }^{+} \mathrm{CD}^{+}$lymphocyte activation after incubation with vemurafenib (one representative patient). Stimulation indexes (SI) were calculated as fold-increase of the CD69 upregulation after vemurafenib stimulation of all four patients compared to control. Kruskal-Wallis test with Dunn's post correction was used to evaluate significances $\left({ }^{*} p<0.05,{ }^{* *} p<0.01,{ }^{* * *} p<0.001\right)$.

Figure 3: Vemurafenib is an AhR antagonist. (A, B), In silico docking of vemurafenib and indirubin to PAS-B homology models (grey). Best docking poses for are shown for vemurafenib (A, van-der-Waals sphere representation with carbons colored in magenta) and indirubin ( $B$, carbons in cyan). Residues of PAS-B forming the cavity are highlighted as stick models. (C), Reporter gene analyses using a XREHepG2 reporter cell line treated with vemurafenib [20; $40 \mu \mathrm{M}$ ] combined with benzo[a]pyrene [2.5 $\mu \mathrm{M}]$. Luciferase activities were offset to protein concentration and normalized to DMSO. (D), Ligand binding was analyzed in guinea pig cytosol, using $2 \mathrm{nM}\left[{ }^{3} \mathrm{H}\right] \mathrm{TCDD}$ in combination with vemurafenib [10; $\left.40 \mu \mathrm{M}\right]$. DNA binding 
was assayed in guinea pig cytosol (E) or using in vitro synthesized AhR/ARNT complexes (F) with TCDD combined with vemurafenib [10; $40 \mu \mathrm{M}]$. Values are normalized to $\left[{ }^{3} \mathrm{H}\right] \mathrm{TCDD}(\mathrm{D})$ or TCDD (E/F). Kruskal-Wallis test with Dunn's post correction was used to evaluate differences $\left({ }^{*} p<0.05\right.$, $\left.{ }^{* *} p<0.01\right)$. (G), AhR translocation into the HaCaT cell nucleus after $2 \mathrm{~h}$ treatment with vemurafenib [100 $\mu \mathrm{M}]$ or in combination with benzo[a]pyrene [2.5 $\mu \mathrm{M}]$. One representative plot of two experiments is displayed showing cytosolic and nucleus fractions together with the total lysate and the controls $\alpha$-tubulin and lamin A/C.

Figure 4: CYP1A1 expression is impaired by vemurafenib in vitro and in vivo. Expression of CYP1A1 mRNA in lesional skin (VIR, $n=4-5)$ compared to healthy controls (HS, $n=11)(A)$, representative immunohistochemistry analysis of CYP1A1 protein in lesional skin versus control $(B)$ and CYP1A1 mRNA expression (C) in primary keratinocytes $(n=14-15)$, skin explants $(n=6)$, and $C D 3^{+} T$ cells $(n=9-15)$. (D) CYP1A1 expression in purified $\mathrm{CD}^{+} \mathrm{T}$ cells treated with vemurafenib alone or in combination with FICZ for $24 \mathrm{~h}(\mathrm{n}=5)$. qPCR-values are shown as fold change relative to $18 \mathrm{~S}$ rRNA expression and compared to normalized DMSO control. (E) Ethoxyresorufin-O-deethylase (EROD) Assay on primary keratinocytes to detect CYP1A1 activity in presence of vemurafenib $(n=4)$. CYP1A1 activity has been adjusted to protein concentration and normalized to DMSO. (F), CD4 ${ }^{+}$T cells $(n=4)$ were treated with vemurafenib $[10 ; 100 \mu \mathrm{M}]$ or dabrafenib [0.1; $1 \mu \mathrm{M}]$. IFNG, IL17A and CYP1A1 gene expression values are shown as fold change normalized to $18 \mathrm{~S}$ rRNA expression compared to DMSO. (G), IFN-y, IL-17 and IL-22 protein expression of $\mathrm{CD}^{+} \mathrm{T}$ cells $(n=4)$ as fold change to DMSO after treatment with vemurafenib or dabrafenib. Data is presented as mean + SEM. Kruskal-Wallis test with Dunn's post correction and Mann-Whitney $U$ test was used to evaluate significant differences $\left({ }^{*} p<0.05,{ }^{* *} p<0.01,{ }^{* * *} p<0.001\right)$.

This article is protected by copyright. All rights reserved. 


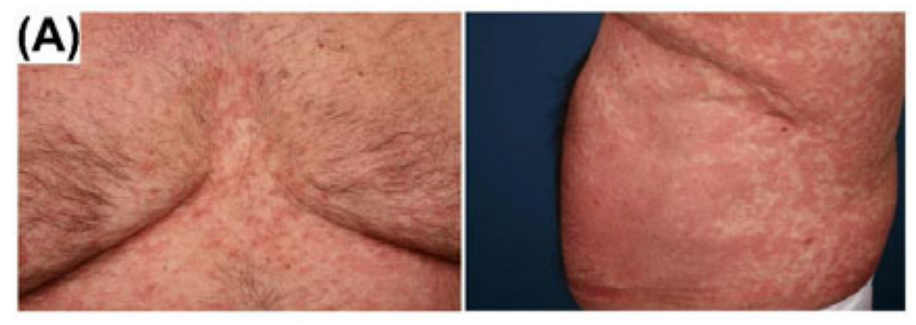

(C)

(B)
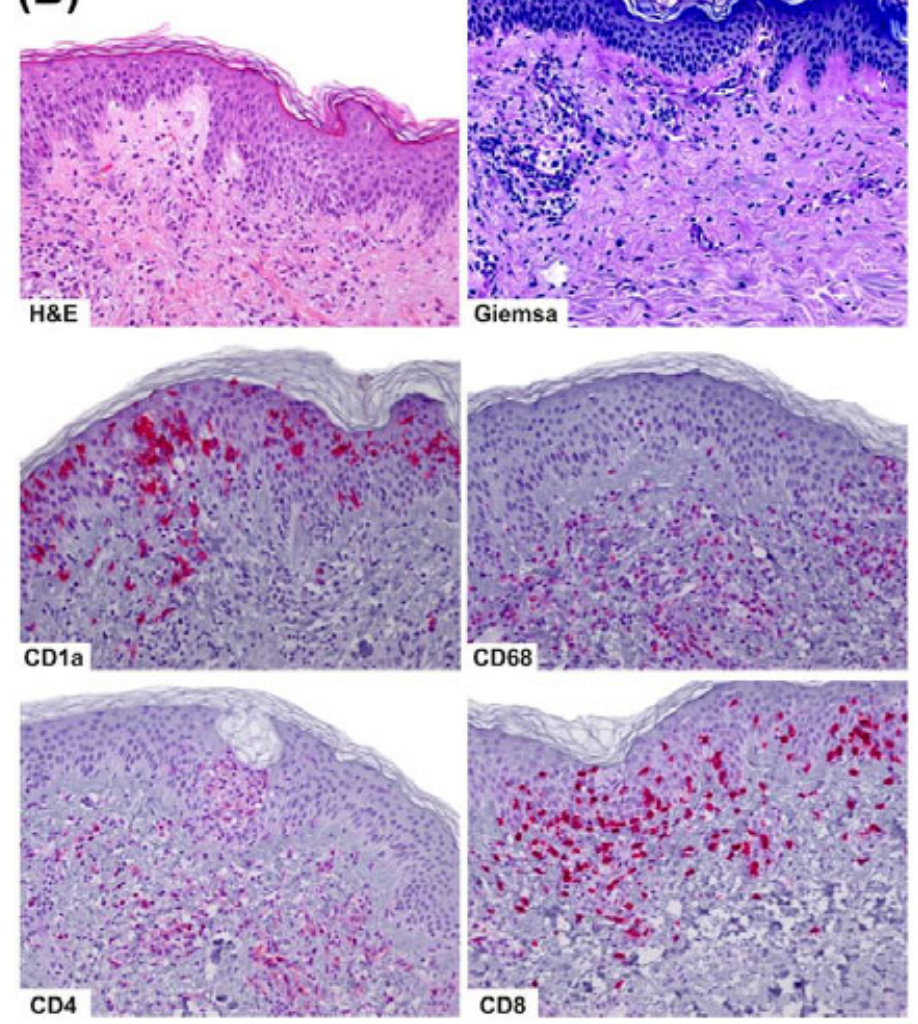
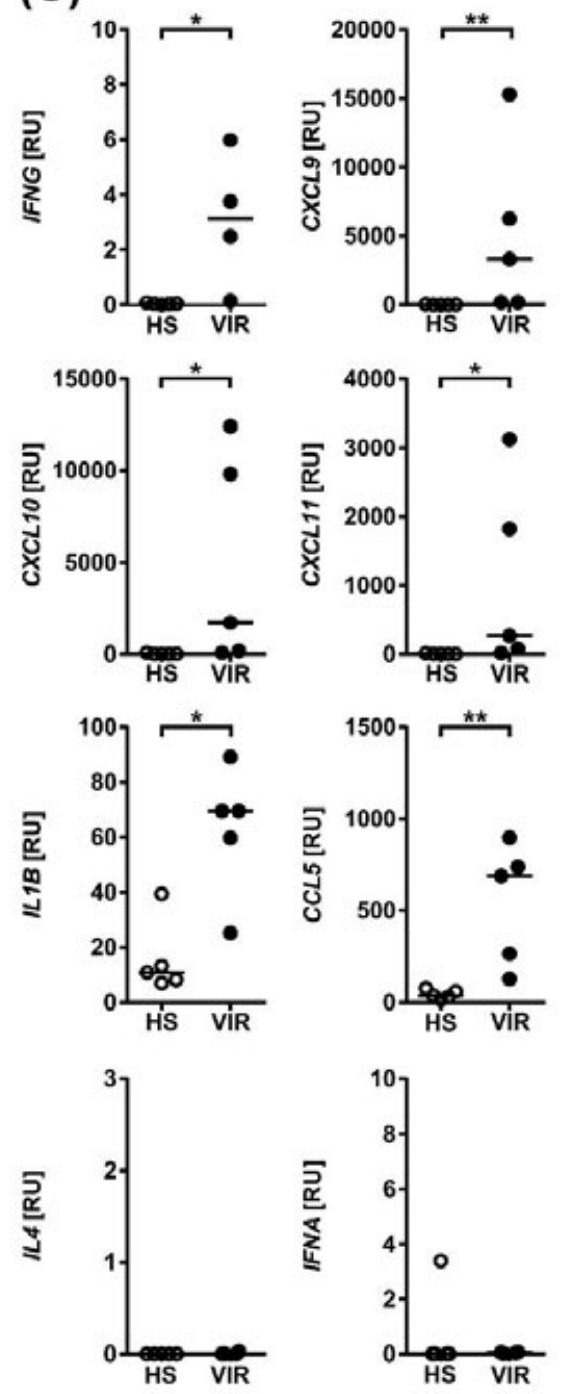

This article is protected by copyright. All rights reserved. 
(A)
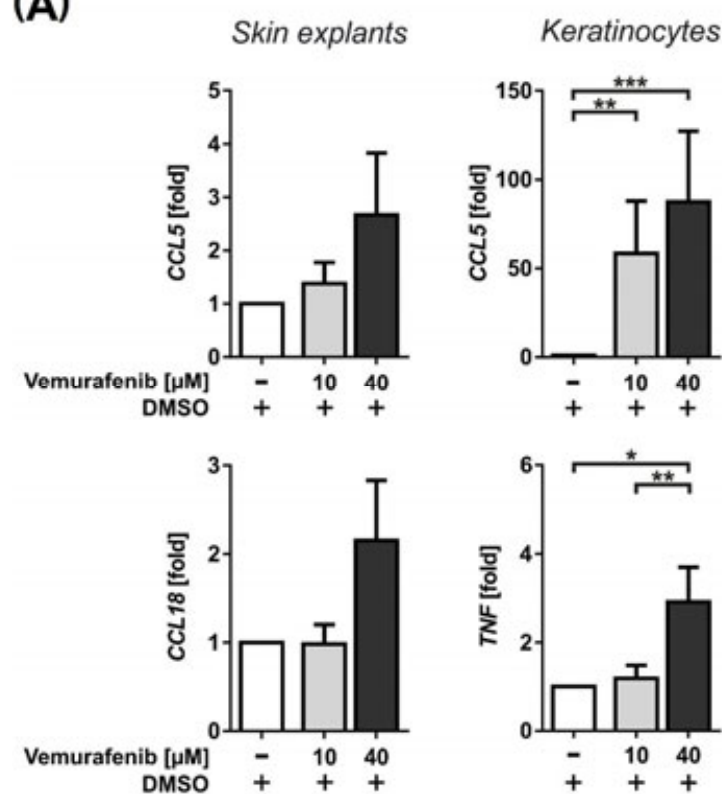

(B)
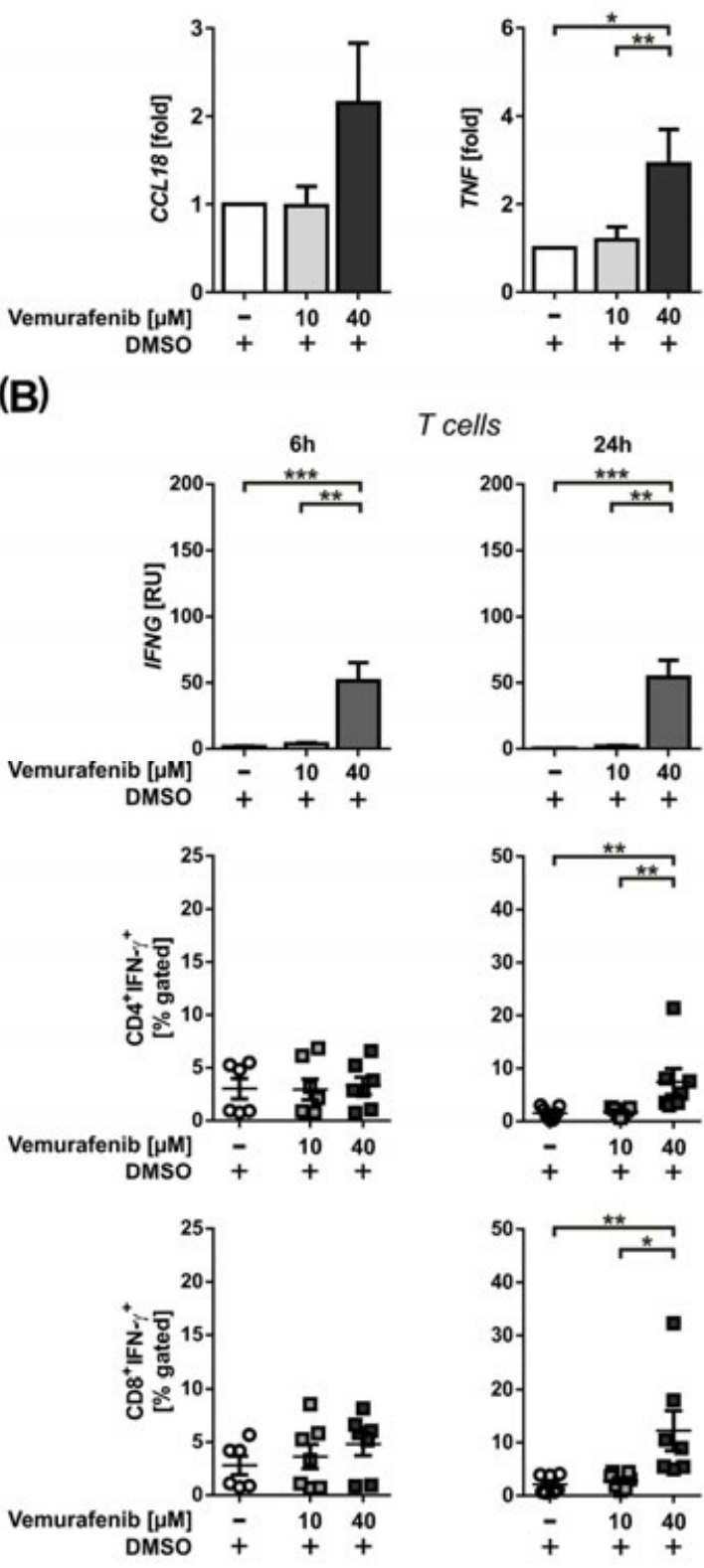

(C)

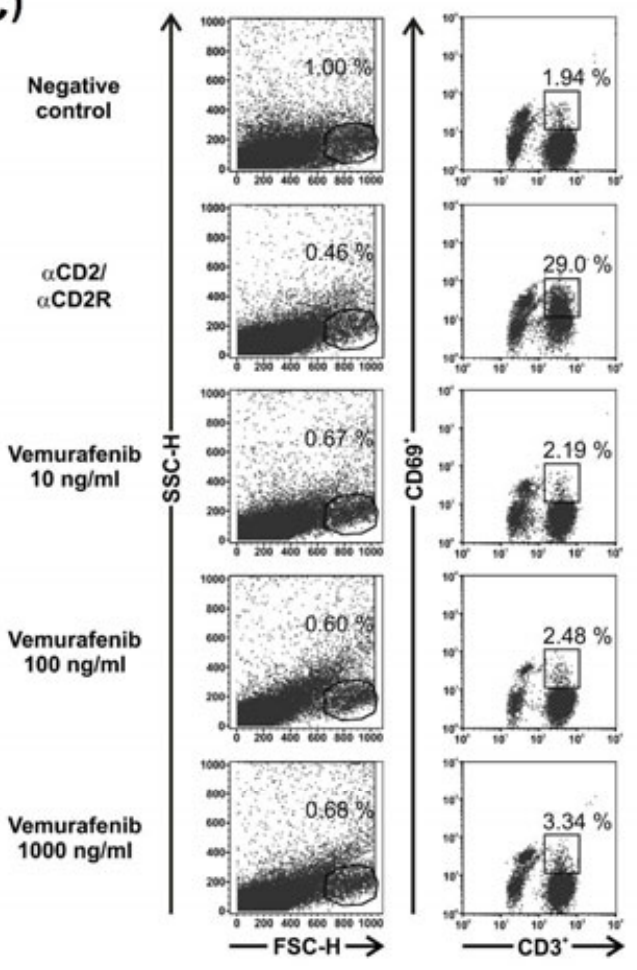

(D)

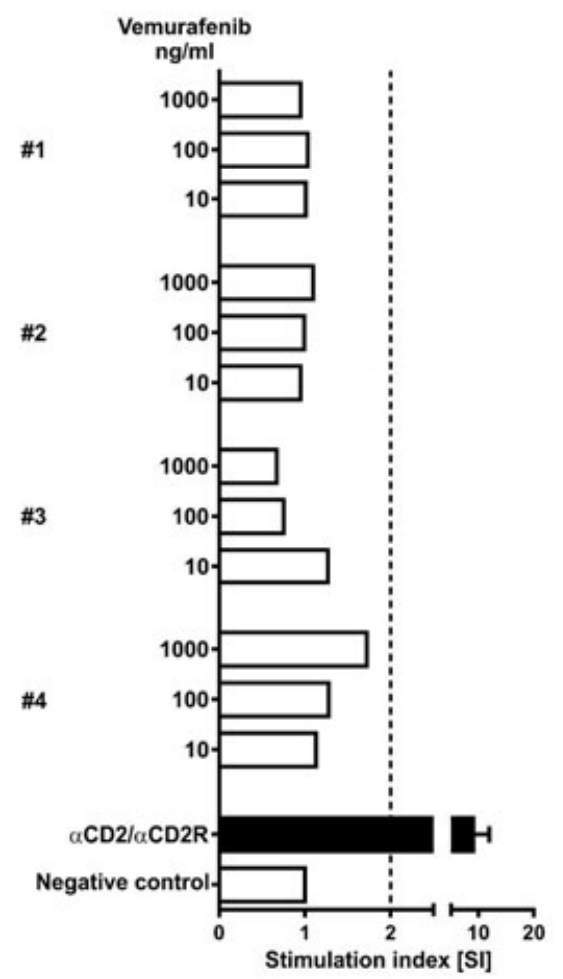

This article is protected by copyright. All rights reserved. 
(A)

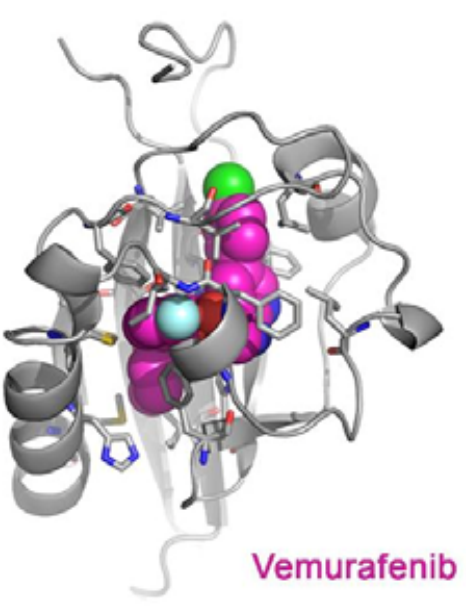

(C)

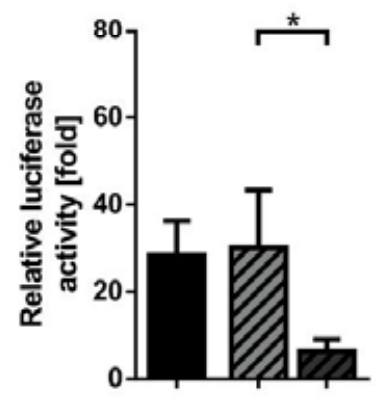

Vemurafenib $[\mu \mathrm{M}] \quad-\quad 2040$ Benzo[a]pyrene $[2.5 \mu \mathrm{M}]++\quad+$

(E)

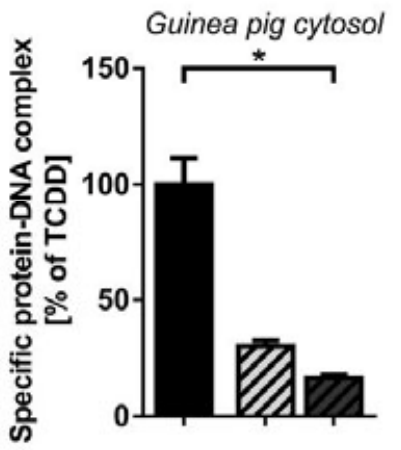

(B)

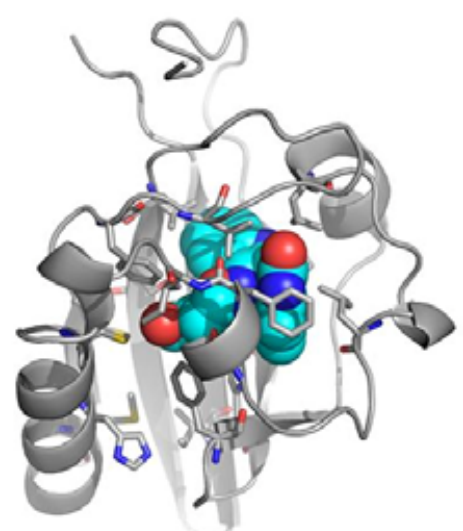

(D)
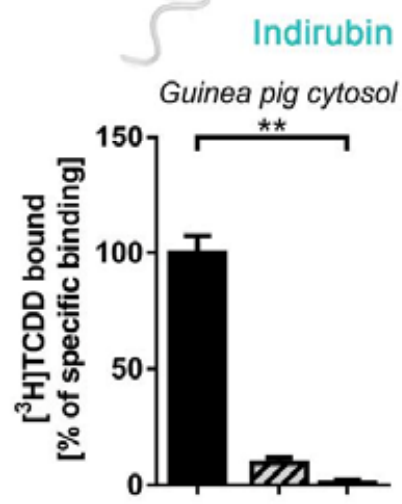

Vemurafenib $[\mu \mathrm{M}] \quad-1040$

[ $\left.{ }^{3} \mathrm{H}\right] \mathrm{TCDD}[2 \mathrm{nM}]++$

(F)

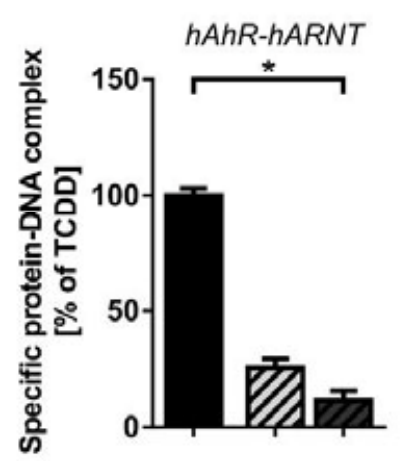

$\begin{array}{r}\text { Vemurafenib }[\mu \mathrm{M}] \\ \operatorname{TCDD}[10 \mathrm{nM}]\end{array}+\quad+\quad+$

Nucleus Total lysate

(G) Cytosol

Vemurafenib $[100 \mu \mathrm{M}]-\quad+\quad+$

Benzo[a]pyrene [2.5 $\mu \mathrm{M}]-++$

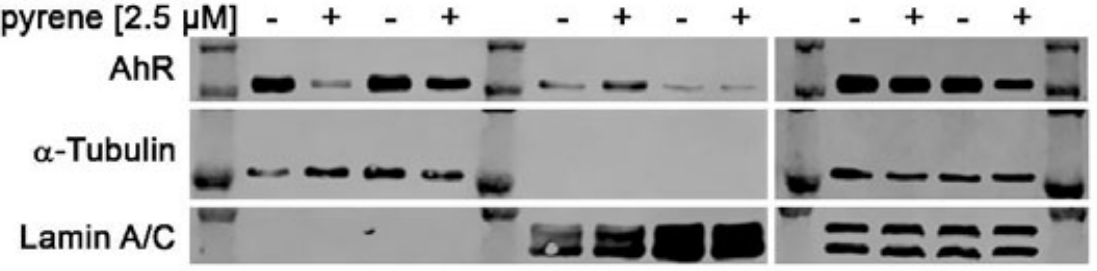


(A)

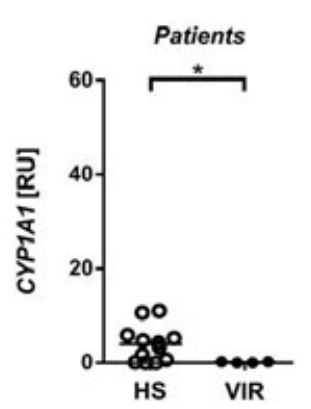

(B)

(C)

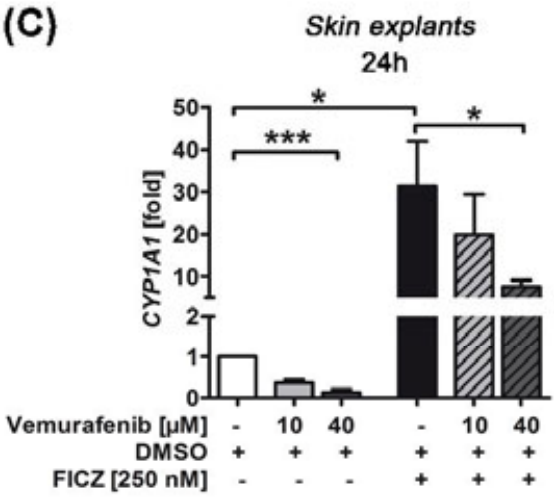

(D)

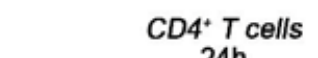

$24 \mathrm{~h}$

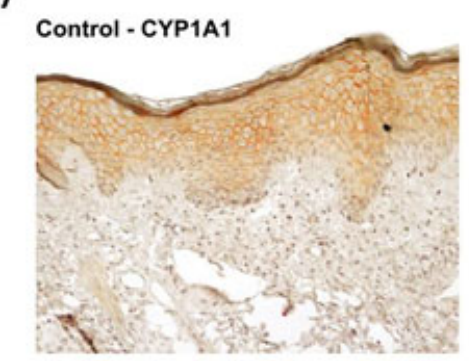

VIR - CYP1A1
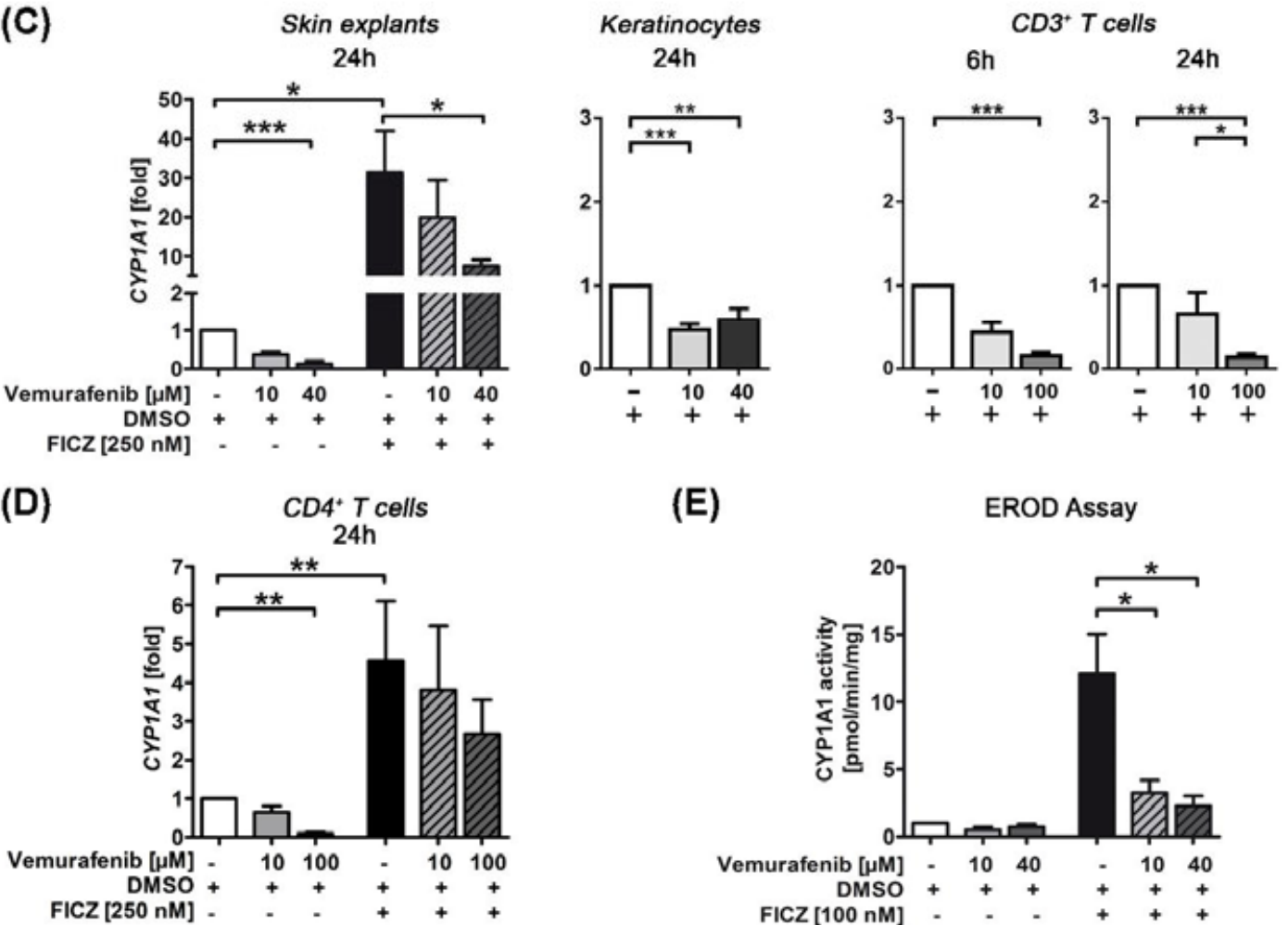

(E)

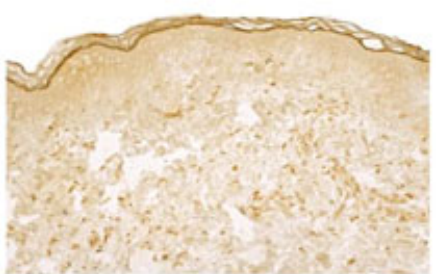

EROD Assay

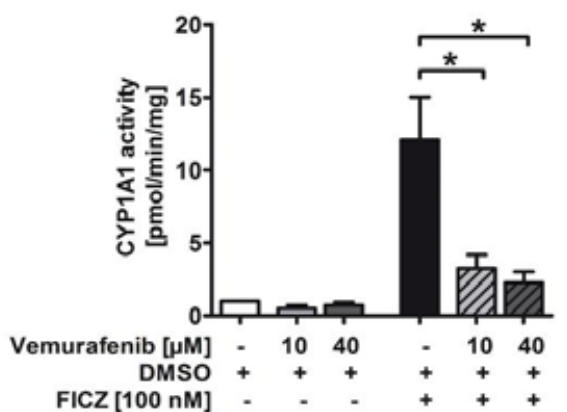

(F)
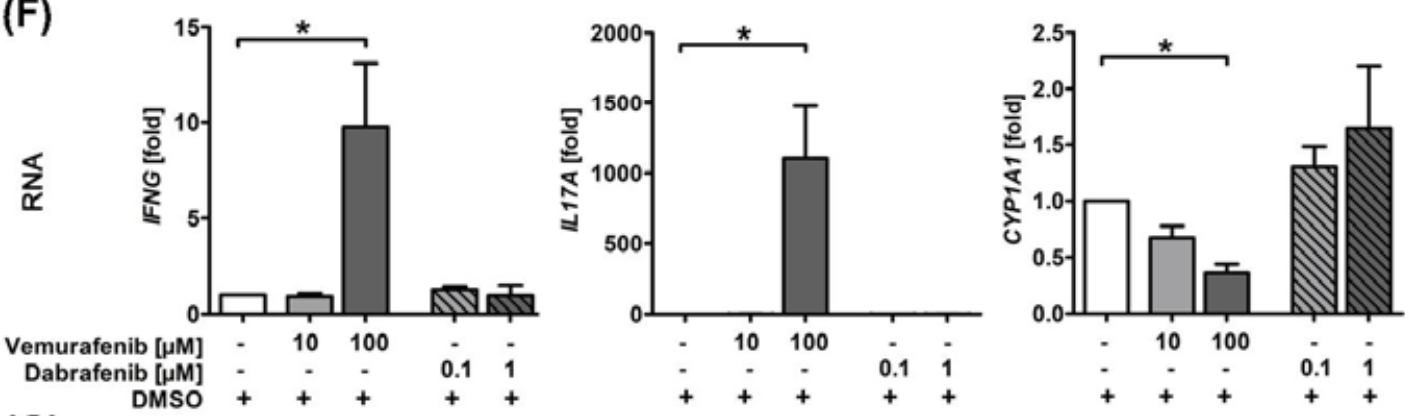

(G)
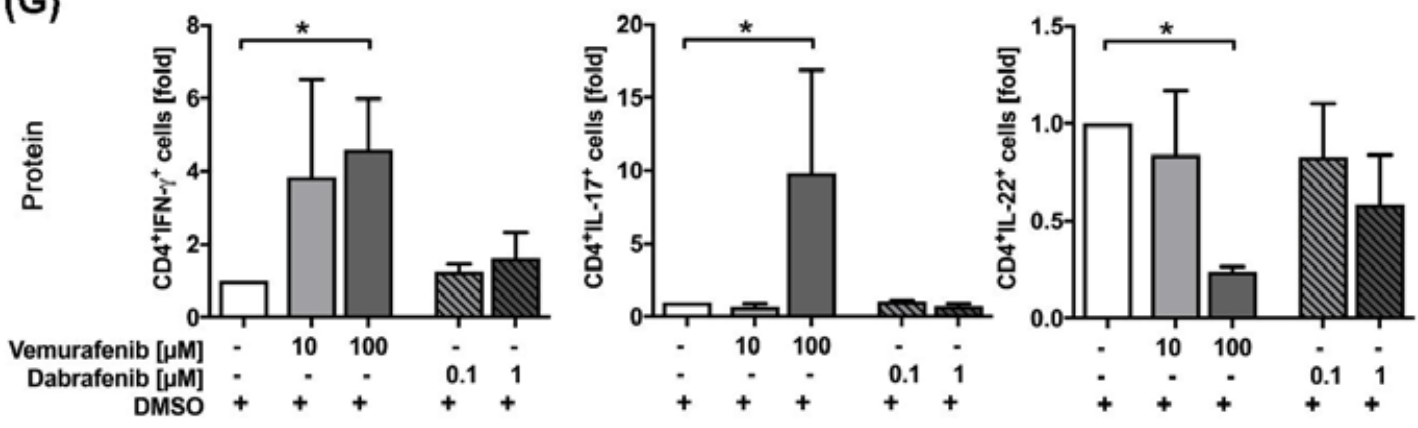

This article is protected by copyright. All rights reserved. 